\title{
Use of Phasors in Nonlinear Analysis
}

\author{
Andrzej Borys and Zbigniew Zakrzewski
}

\begin{abstract}
In this paper, the well-known method of phasor analysis of linear ac circuits is extended in a rigorous mathematical way to nonlinear analysis. This fills the lack of such a theory in the literature. The results derived enable carrying out the needed corrections of some results published recently that regard harmonic distortion analysis of weakly nonlinear circuits.
\end{abstract}

Keywords-phasor, Volterra series, analysis of nonlinear AC circuits, harmonic distortion analysis of weakly nonlinear circuits, balance of harmonics

\section{INTRODUCTION}

$\mathbf{I}$ $\mathrm{N}$ a whole series of papers [1]-[10] published in the last decade, S. O. Cannizarro, G. Palumbo, S. Pennisi, and M. Pennisi have created a new method of calculation of harmonic distortion in mildly nonlinear circuits, and named it "an approach exploiting the phasor notation in the frequency domain" (see [10]). In all papers mentioned above, they have argued that their approach is an alternative to the (too much complicated in opinion of some researchers) Volterra series method, traditionally used in nonlinear distortion analysis of weakly nonlinear circuits [11]-[13] (we cite at this point only a few papers, pioneers of application of the Volterra series in the area of nonlinear circuits and systems). As it will be evident in the course of this paper, their method is neither new nor alternative to the Volterra series approach.

S. O. Cannizarro, G. Palumbo, S. Pennisi, and M. Pennisi claim that the roots of their approach lie, first of all, in the phasor analysis of linear circuits driven by ac signals (sinusoidal signals). This basic and nowadays elementary means of analysis of ac circuits was found and developed mostly by O. Heaviside and Ch. P. Steinmetz - two very famous electrical engineers of that time - more than one hundred years ago. Ch. P. Steinmetz has continuously promoted this method [14] in the United States of America. In Poland, B. Konorski has written an excellent textbook [15] promoting the above approach.

Second, S. O. Cannizarro et al. say that their method relies upon balancing the flows of harmonics in nodes of a nonlinear circuit (or of its representing graph).

In none of the papers [1]-[10], the approach discussed was developed systematically, thoroughly, consequently stepby-step according to some mathematical rigour. This lack of appropriate carefulness is the reason of occurrence of many errors and faults, of different kinds, in the aforementioned papers. For example, an operator "o" in [2] was introduced fully ad hoc, without any explanation. In consequence, its

The authors are with the Institute of Telecommunications, Faculty of Telecommunications and Electrical Engineering, University of Technology and Life Sciences (UTP), S. Kaliskiego 7, 85-789 Bydgoszcz, Poland (e-mails: \{andrzej.borys; zbizak\}@utp.edu.pl). descriptive definition in [2] is not correct; we show its incorrectness in Section II.

The objective of this paper is to show how the phasors, known from the classical phasor analysis developed for linear ac circuits by $\mathrm{O}$. Heaviside and Ch. P. Steinmetz, can be applied and exploited correctly in the nonlinear analysis. The detailed and complete derivations are presented here.

The remainder of the paper is organized as follows. In Section II, basics of phasor analysis formulated for linear ac circuits are recalled. In the next section, the rules, principles, derivations, and expressions of this analysis are consequently extended to the nonlinear analysis, using the needed mathematical rigour. The method of phasor nonlinear analysis is summarized in the Section IV, by listing its main characteristic features.

\section{Phasor Principles in AnAlysis of Linear AC CIRCUITS}

Before beginning discussion of the problem of correct application of phasors in nonlinear analysis, we have first to recall the needed here basics of phasor analysis formulated for linear ac circuits. These fundamentals are summarized in what follows.

We remind once again that the phasor analysis (called also the symbolic analysis in some papers and textbooks), as formulated by $\mathrm{O}$. Heaviside and Ch. P. Steinmetz and exploited widely afterwards, applies only to linear circuits driven by sinusoidal signals. In this form, it is obviously not suitable for nonlinear circuits. Our task in this paper is to extend this approach to a class of nonlinear circuits called weakly (or mildly) nonlinear ones [1]-[3], [11]-[13] driven by a single sinusoidal (harmonic) signal.

We distinguish here between rotating (alternating) and nonrotating (non-alternating) phasors. To this end, we define the rotating phasor as

$$
A e^{j \omega t}=\left(|A| e^{j \varphi_{A}}\right) e^{j \omega t}
$$

where its complex amplitude $A=|A| e^{j \varphi_{A}}$ is in general a complex number having magnitude $|A|$ and phase $\varphi_{A}$ in polar representation and may depend upon $\omega$; however, $A$ is assumed to be independent of the time variable $t$. The variable $\omega=2 \pi f$ means an angular frequency with $f$ used for denoting the usual frequency variable. Moreover, $j=\sqrt{-1}$ in (1).

Note from (1) that when the value of time variable $t$ increases the phasor rotates counter-clockwise on the complex $z$ plane around the point $(0,0)$ of this plane. In other words, interpreting the phasor as a vector, we can say that the vector given by (1) is such a vector of the constant length $|A|$ that rotates around the point $(0,0)$ when the value of time variable $t$ 
increases. Moreover, it is seen from (1) that $A e^{j \omega t}$ represents a complex harmonic signal of frequency $f$. Note also that (1) can be rewritten as

$$
\begin{gathered}
a(t)=|A| e^{j\left(\omega t+\varphi_{A}\right)}= \\
=|A|\left(\cos \left(2 \pi f+\varphi_{A}\right)+j \sin \left(2 \pi f+\varphi_{A}\right)\right)
\end{gathered}
$$

The form given by (2) and the relationships $\operatorname{Re}\{z\}=\left(z+z^{*}\right) / 2$ and $\operatorname{Im}\{z\}=\left(z+z^{*}\right) /(2 j)$, where $\operatorname{Re}\{z\}$ and $\operatorname{Im}\{z\}$ mean the real and imaginary parts of a complex number $z$, respectively, with “*” denoting the complex conjugate value of $z$, allow to express the real ac quantities (signals) $|A| \cos \left(2 \pi f+\varphi_{A}\right)$ and $|A| \sin \left(2 \pi f+\varphi_{A}\right)$ through complex numbers (complex harmonic signals) of the form given by (1).

Let us now divide the rotating phasor given by (1) by $e^{j \omega t}$. As a result, we get a non-rotating phasor. It is equal in this case to the complex number $A$ from (1).

Linear analog circuits consist of such components as linear resistors, inductors, capacitors, and linear amplifying (or attenuating) elements. These components may be connected to each other in different ways; the points of connection of their terminals are called nodes. In these nodes, the signals are added to or subtracted from. These operations (addition and subtraction) do not change, however, the form of the rotating phasors does because $A e^{j \omega t} \pm B e^{j \omega t}=(A \pm B) e^{j \omega t}$ holds. We see that only their amplitudes change (in the above example, from $A$ or $B$ to $A \pm B$. In other words, the non-rotating phasors, which are complex numbers independent of time, are added to or subtracted from. And this is the basic principle of the usage of phasors in the so-called phasor analysis (symbolic analysis) of linear ac circuits.

Recapitulating the last point: analysis of a linear ac circuit is in fact carried out exclusively with the use of non-rotating phasors - some authors say that it is done in the so-called frequency domain, $j f=j \omega /(2 \pi)$. This leads to getting the resulting phasor in this domain. Obtaining the final result in the time domain needs only one operation more, namely, multiplication of the resulting non-rotating phasor by a complex time function $e^{j 2 \pi f t}$. The latter operation gives the final result, as a rotating phasor, in the time domain. Moreover, note also that it follows from the above that we can interpret the calculations using only the non-rotating phasors as constituting a "purely" frequency domain method, but the one applying rotating phasors as a "mixed" method that combines the frequency domain with the time domain results.

We also remind in this section the fact that the phasor analysis of linear ac circuits applies only to the steady state of these circuits - the transient behaviour of these circuits is omitted in this analysis. Furthermore, we remember the standard and basic result saying that when a linear circuit is driven by a signal of the form given by (1) (single complex harmonic signal) then all the voltages and currents (signals) in it have the same form: of a complex number (eventually dependent upon $f$ ) multiplied by a time function $e^{j 2 \pi f t}$. This means that other steady state signal components of harmonics different from $f$ cannot appear in such a circuit. (Note that this result was exploited in discussion of the previous point.)
The fact that "the steady state higher harmonic" cannot be generated in linear ac circuits can be derived from their linearity property. We show this now shortly, without going into too many mathematical details. To this end, we recall first that any of the possible basic linear components (as for example, linear resistor, linear inductor, and linear capacitor) as well as the whole circuit possess their descriptions in form of the linear operator having (excluding possible pathological cases [16], [17]) the one-dimensional convolution integral representation as

$$
y(t)=\int_{-\infty}^{\infty} h(\tau) x(t-\tau) d \tau
$$

where $y(t)$ and $x(t)$ denote component (circuit) output and input signal, respectively. Moreover, $h(t)$ in (3) is the socalled element (circuit) impulse response. Numerous examples of it for such linear circuits as analog filters, amplifiers, etc. can be found in papers and textbooks. On the other hand, as the basic circuit elements: linear resistor, linear inductor, and linear capacitor are concerned, we can express their impulse responses as follows:

For resistor,

$$
h_{R}(t)=R \delta(t)
$$

on assuming $x(t)$ and $y(t)$ in (3) to mean the current $i_{R}(t)$ flowing through the resistor of resistance $R$ and the voltage across this resistor $v_{R}(t)$, respectively, or

$$
h_{G}(t)=G \delta(t)
$$

when applying the opposite convention as above with regard to $x(t)$ and $y(t)$, with $G$ meaning the conductance of the resistor considered, i.e. $G=1 / R . \delta(t)$ in (4a) and (4b) means the Dirac impulse.

Further, for inductor,

$$
h_{L}(t)=G \delta^{\prime}(t)
$$

on assuming for this case $x(t)$ and $y(t)$ in (3) to mean the current $i_{L}(t)$ flowing through the inductor of inductance $L$ and the voltage $v_{L}(t)$ across this inductor, respectively, or

$$
h_{1 / L}(t)=L^{-1} 1(t)=\left\{\begin{array}{cc}
\frac{1}{L} & t \geq 0 \\
0 & t<0
\end{array}\right.
$$

when applying the opposite convention as above with regard to $x(t)$ and $y(t) . \delta^{\prime}(t)$ in (5a) means the derivative of the Dirac impulse $\delta(t)$. Moreover, $1(t)$ in (5b) stays for the Heaviside unit step function.

And finally, for capacitor,

$$
h_{C}(t)=C^{-1} 1(t)=\left\{\begin{array}{cc}
\frac{1}{C} & t \geq 0 \\
0 & t<0
\end{array}\right.
$$

on assuming for this case $x(t)$ and $y(t)$ in (3) to mean the voltage $v_{c}(t)$ occurring between the capacitor terminals (across the capacitor) and the current $i_{c}(t)$ flowing through this capacitor of capacitance $C$, respectively, or

$$
h_{1 / C}(t)=C \delta^{\prime}(t)
$$

when applying the opposite convention as above regarding the meaning of $x(t)$ and $y(t)$. 
All the impulse responses given by $(4 a)-(6 b)$ can be easily derived using the following definition of the impulse response: it is the response of an circuit element or a whole circuit to the input signal in form of the Dirac impulse $\delta(t)$. So applying this definition in the relations: $v_{R}(t)=R i_{R}(t)$,

$$
\begin{gathered}
i_{R}(t)=1 / R v_{R}(t), \quad v_{L}(t)=L d i_{L}(t) / d t \\
i_{L}(t)=1 / L \int_{-\infty}^{t} v_{L}(\tau) d \tau, \quad v_{C}(t)=1 / C \int_{-\infty}^{t} i_{C}(\tau) d \tau \\
\text { and } i_{C}(t)=C d v_{C}(t) / d t,
\end{gathered}
$$

we get successively (4a) - (6b) .

Second, let us now apply the input signal of the form given by (1), i.e. $x(t)=X e^{j 2 \pi f t}$ in (3). We arrive then at

$$
\begin{gathered}
y(t)=\int_{-\infty}^{\infty} h(\tau) X e^{j 2 \pi f(t-\tau)} d \tau= \\
=H(f) X e^{j 2 \pi f t}=H(f) x(t)
\end{gathered}
$$

with $H(f)$ being the Fourier transform of $h(t)$, that is

$$
H(f)=\int_{-\infty}^{\infty} h(\tau) e^{-j 2 \pi f \tau} d \tau
$$

The result given by (7a) proves that an ac circuit and its elements do not generate (steady state) signal components possessing higher harmonics of the input frequency $f$.

Furthermore, (7a) shows that the non-rotating phasor in phasor analysis of linear ac circuits is equal to the Fourier transform of the corresponding impulse response multiplied by the complex signal amplitude, with one possible exception regarding the Heaviside unit step function $1(t)$ occurring in (5b) and (6a). We will discuss this case separately. Before doing this, however, we draw the reader's attention to the fact that $H(f)$ itself (given by (7b)), multiplying the complex amplitude $X$ to result in the non-rotating phasor $H(f) X$, is also called the non-rotating phasor in the literature. Therefore, in this paper, to avoid any inconsequences, we distinguish between the non-rotating phasors containing the signal complex amplitudes of the first, second, and third harmonics, or the products of these harmonics amplitudes - and those ones dependent exclusively upon the parameters of circuit elements. We name the first group the non-rotating phasors of first type (being in fact the multidimensional Fourier transforms of the amplitudes of harmonic components of currents and voltages occurring in the circuit). And consequently, we name the nonrotating phasors independent of the harmonics amplitudes, the non-rotating phasors of the second type (being admittances, impedances, transfer functions formulated in the frequency domain, etc.). In what follows, the type of a given non-rotating phasor will clearly follow from the context.

Using (7b) in particular cases of the impulse responses given by (4a), (4b), (5a), and (6b), we get the well-known results: $R$, $G, j 2 \pi f L$, and $j 2 \pi f C$, respectively. Furthermore, we arrive at $1 /(j 2 \pi f L)+1 / 2 \cdot \delta(f)$ and $1 /(j 2 \pi f C)+1 / 2 \cdot \delta(f)$ in the remaining cases of $(5 b)$ and $(6 a)$, respectively.

The non-rotating phasors for linear inductor and capacitor are derived in the literature with the use of equations $v_{L}(t)=L d i_{L}(t) / d t$ and $i_{C}(t)=C d v_{C}(t) / d t$, respectively.
Note further that on assuming in these equations the following form of the signal $i_{L}(t)$ or $v_{C}(t): A e^{j 2 \pi f t}$ (in accordance with (1)), we get

$$
v_{L}(t)=L \frac{d}{d t}\left(A e^{j 2 \pi f t}\right)=j 2 \pi f L i_{L}(t)
$$

or

$$
i_{C}(t)=C \frac{d}{d t}\left(A e^{j 2 \pi f t}\right)=j 2 \pi f C v_{C}(t) .
$$

Rearranging the signals (voltages and currents) in equations (8a) and (8b), we can write

$$
i_{L}(t)=\frac{1}{j 2 \pi f L} v_{L}(t) \Rightarrow \frac{i_{L}(t)}{v_{L}(t)}=\frac{1}{j 2 \pi f L}
$$

and

$$
v_{C}(t)=\frac{1}{j 2 \pi f C} i_{C}(t) \Rightarrow \frac{v_{C}(t)}{i_{C}(t)}=\frac{1}{j 2 \pi f C}
$$

Assuming now that $v_{L}(t)$ and $i_{C}(t)$ in (9a) and (9b), respectively, stand for the input signals, but the signals $i_{L}(t)$ and $v_{C}(t)$ for the output ones, it follows that the corresponding non-rotating phasors are equal to $1 /(j 2 \pi f L)$ and $1 /(j 2 \pi f C)$, respectively. So they differ from those derived using the convolution integral representation by a component $1 / 2 \cdot \delta(t)$. However, see that the latter component equals zero for frequencies $f \neq 0$. Hence, in fact, the previous and the latter expressions are identical for all the frequencies different from $f=0$ (direct current, dc). But for analyzing dc circuits, we do not need to use phasor method. Then, we can use one of the approaches applicable to purely resistive circuits (in which, if capacitors occur, they are treated as open circuit elements, but inductors as short-circuited elements).

\section{BASIC RULES FOR USE OF PHASORS IN ANALYSIS OF NONLINEAR CIRCUITS}

It is well known that a natural extension of the one-dimensional convolution integral (3) to description of the steady state of nonlinear circuits is the Volterra series [11]-[13]

$$
\begin{gathered}
y(t)=y^{(1)}(t)+y^{(2)}(t)+y^{(3)}(t)+\cdots= \\
=\int_{-\infty}^{\infty} h^{(1)}(\tau) x(t-\tau) d \tau+ \\
+\int_{-\infty}^{\infty} \int_{-\infty}^{\infty} h^{(2)}\left(\tau_{1}, \tau_{2}\right) x\left(t-\tau_{1}\right) x\left(t-\tau_{2}\right) d \tau_{1} d \tau_{2}+ \\
+\int_{-\infty}^{\infty} \int_{-\infty}^{\infty} \int_{-\infty}^{\infty} h^{(3)}\left(\tau_{1}, \tau_{2}, \tau_{3}\right) . \\
\cdot x\left(t-\tau_{1}\right) x\left(t-\tau_{2}\right) x\left(t-\tau_{3}\right) d \tau_{1} d \tau_{2} d \tau_{3}+\ldots
\end{gathered}
$$

In (10), $y(t)$ and $x(t)$ mean the output and input signal, respectively, at a nonlinear circuit. Moreover, the terms $y^{(1)}(t)$, $y^{(2)}(t), y^{(3)}(t)$, and so on, which are components of $y(t)$, are called the circuit partial responses of the corresponding orders: first, second, third, and of higher orders, accordingly. Furthermore, $h^{(1)}(\tau), h^{(2)}\left(\tau_{1}, \tau_{2}\right), h^{(3)}\left(\tau_{1}, \tau_{2}, \tau_{3}\right)$, and so on, are, respectively, the first order, second order, third order, and so on, nonlinear impulse responses of the circuit considered. Finally, we remark that $y^{(1)}(t)$ and $h^{(1)}(\tau)$ are also called 
circuit's linear partial response and its linear impulse response, respectively.

For the so-called weakly (mildly) nonlinear circuits, the series given by (10) is truncated to the first three components. Note that the above constitutes, at the same time, the definition of the aforementioned class of circuits.

Observe further that the truncated Volterra series similarly as (3), which was assumed in the previous section to describe any linear circuit component or whole linear circuit, can be applied for description of all possible nonlinear circuit elements and whole nonlinear circuits (of the class defined above). Therefore, in what follows, we will restrict ourselves to just this general form of description.

Applying now the input signal $x(t)=X e^{j 2 \pi f t}$ in the truncated to the first three components Volterra series given by (10), we arrive at

$$
\begin{gathered}
y(t) \cong \hat{y}(t)=y^{(1)}(t)+y^{(2)}(t)+y^{(3)}(t)= \\
=\int_{-\infty}^{\infty} h^{(1)}(\tau) X e^{j 2 \pi f(t-\tau)} d \tau+ \\
+\int_{-\infty}^{\infty} \int_{-\infty}^{\infty} h^{(2)}\left(\tau_{1}, \tau_{2}\right) X^{2} e^{j 2 \pi f\left(t-\tau_{1}\right)} e^{j 2 \pi f\left(t-\tau_{2}\right)} d \tau_{1} d \tau_{2}+ \\
+\int_{-\infty}^{\infty} \int_{-\infty}^{\infty} \int_{-\infty}^{\infty} h^{(3)}\left(\tau_{1}, \tau_{2}, \tau_{3}\right) \cdot \\
\cdot X^{3} e^{j 2 \pi f\left(t-\tau_{1}\right)} e^{j 2 \pi f\left(t-\tau_{2}\right)} e^{j 2 \pi f\left(t-\tau_{3}\right)} d \tau_{1} d \tau_{2} d \tau_{3}= \\
=X e^{j 2 \pi f t} \cdot \int_{-\infty}^{\infty} h^{(1)}(\tau) e^{-j 2 \pi f \tau} d \tau+ \\
+X^{2} e^{j 2 \pi(2 f) t} \cdot \int_{-\infty}^{\infty} \int_{-\infty}^{\infty} h^{(2)}\left(\tau_{1}, \tau_{2}\right) e^{-j 2 \pi f \tau_{1}} e^{-j 2 \pi f \tau_{2}} d \tau_{1} d \tau_{2}+ \\
+X^{3} e^{j 2 \pi(3 f) t} \cdot \int_{-\infty}^{\infty} \int_{-\infty}^{\infty} \int_{-\infty}^{\infty} h^{(3)}\left(\tau_{1}, \tau_{2}, \tau_{3}\right) \cdot \\
\cdot e^{-j 2 \pi f \tau_{1}} e^{-j 2 \pi f \tau_{2}} e^{-j 2 \pi f \tau_{3}} d \tau_{1} d \tau_{2} d \tau_{3}
\end{gathered}
$$

The symbol "^" above $y(t)$ in (11) means operation of truncation of the Volterra series given by $y(t)$ to only the first three components (in other words, taking into account only the first three orders of nonlinearity in a circuit).

Introducing then the following multidimensional Fourier transforms (defined for example in [13]) of the nonlinear impulse responses of the first, second, and third order, $h^{(1)}(\tau)$, $h^{(2)}\left(\tau_{1}, \tau_{2}\right)$, and $h^{(3)}\left(\tau_{1}, \tau_{2}, \tau_{3}\right)$, respectively

$$
\begin{gathered}
H^{(1)}(f)=\int_{-\infty}^{\infty} h^{(1)}(\tau) e^{-j 2 \pi f \tau} d \tau \\
H^{(2)}(f, f)=\int_{-\infty}^{\infty} \int_{-\infty}^{\infty} h^{(2)}\left(\tau_{1}, \tau_{2}\right) e^{-j 2 \pi f \tau_{1}} e^{-j 2 \pi f \tau_{2}} d \tau_{1} d \tau_{2} \\
H^{(3)}(f, f, f)=\int_{-\infty}^{\infty} \int_{-\infty}^{\infty} \int_{-\infty}^{\infty} h^{(3)}\left(\tau_{1}, \tau_{2}, \tau_{3}\right) . \\
\cdot e^{-j 2 \pi f \tau_{1}} e^{-j 2 \pi f \tau_{2}} e^{-j 2 \pi f \tau_{3}} d \tau_{1} d \tau_{2} d \tau_{3}
\end{gathered}
$$

into (11), we obtain

$$
\hat{y}(t)=H^{(1)}(f) X e^{j 2 \pi f t}+H^{(2)}(f, f) X^{2} e^{j 2 \pi(2 f) t}+
$$

$$
\begin{gathered}
+H^{(3)}(f, f, f) X^{3} e^{j 2 \pi(3 f) t}= \\
=H^{(1)}(f) x(t)+H^{(2)}(f, f)(x(t))^{2}+ \\
+H^{(3)}(f, f, f)(x(t))^{3} .
\end{gathered}
$$

The result given by (13) shows that the resulting rotating phasor $\hat{y}(t)$ in the nonlinear analysis using phasors (and the Volterra series that is truncated to first three components) is a sum of three rotating phasors $y^{(1)}(t), y^{(2)}(t)$, and $y^{(3)}(t)$, which rotate with the different angular frequencies $\omega=2 \pi f$, $2 \omega=2 \pi(2 f)$, and $3 \omega=2 \pi(3 f)$, respectively. Further, the non-rotating phasors associated with the rotating ones mentioned above are equal to $H^{(1)}(f) X, H^{(2)}(f, f) X^{2}$, and $H^{(3)}(f, f, f) X^{3}$, respectively.

Let us rewrite now (13) in the following form

$$
\begin{gathered}
\hat{y}(t)=Y(f, t)=\left[H^{(1)}(f)+H^{(2)}(f, f)(x(t))+\right. \\
\left.+H^{(3)}(f, f, f)(x(t))^{2}\right] x(t) .
\end{gathered}
$$

In (14), $Y(f, t)$ is used to write in a shorter form the following: $H^{(1)}(f)+H^{(2)}(f, f)(x(t))+H^{(3)}(f, f, f)(x(t))^{2}$. Observe that the expression (14) allows us to interpret the resulting rotating phasor $\hat{y}(t)$ as the rotating phasor $x(t)$ modulated in amplitude by a time function dependent upon $x(t)$. This evidently results from the form of $Y(f, t)$ in (14). However, the above observation has no practical importance for carrying out calculations.

In papers [1]-[10], the coefficients $H^{(1)}(f), H^{(2)}(f, f)$ and $H^{(3)}(f, f, f)$ occurring in the representation given by (13) have been named nonlinear coefficients, without identifying any connection with the Volterra series. Here, we see that they are multidimensional Fourier transforms of the nonlinear impulse responses of the corresponding orders. These transforms are called also (see, for example [13]) the nonlinear transfer functions of the corresponding orders (of the first (representing the linear transfer function), second, and third order, respectively).

Notational remark: In [1]-[10], the nonlinear coefficients $H^{(1)}(f)=a_{1}(j \omega), H^{(2)}(f, f)=a_{2}(j \omega)$ and $H^{(3)}(f, f, f)=a_{3}(j \omega)$ with $j \omega=2 \pi f$ have been denoted as indicated (i.e. as $a_{1}(j \omega), a_{2}(j \omega)$, and $a_{3}(j \omega)$, successively). That is as functions of only one variable $\omega$ (or $f$ ). This led, however, to occurrence of some errors in calculations presented in [1]-[10]: for example in equation (19) in [1] $a_{2}^{2}(j \omega)$ stands for $H^{(2)}(f, f) \cdot H^{(2)}(f, 2 f)$. It was assumed therein that the following holds: $H^{(2)}(f, f)=H^{(2)}(f, 2 f)$, but obviously generally this is not true. So the aforementioned notational simplification, as leading to errors, should not be exploited in calculations.

The general relation (13) derived for the nonlinear phasor analysis of ac circuits is not only counterpart of the corresponding relation (7) in the phasor analysis of linear ac circuits. Once again, we point out that it holds exclusively when the input signal applied to the given circuit element or the whole circuit is of the form $x(t)=X e^{j 2 \pi f t}$ (a complex single harmonic signal). However, in nonlinear ac circuits, as it is well-known (see, for example, [13], [18]), the signal components at harmonics $2 f, 3 f$, and higher ones of the 
signal $x(t)=X e^{j 2 \pi f t}$ occur at circuit elements, too. Then, obviously, (13) cannot be used and we need another formula. In what follows now, we derive this formula that will constitute second counterpart of (7) needed in the phasor analysis of nonlinear ac circuits. To this end, we assume the following form of the signal $x(t)$

$$
x(t)=X_{f} e^{j 2 \pi f t}+X_{2 f} e^{j 2 \pi(2 f) t}+X_{3 f} e^{j 2 \pi(3 f) t}
$$

to apply in the truncated series (10). In (15), $X_{f}, X_{2 f}$, and $X_{3 f}$ denote the complex amplitudes of the signal components at harmonics $f, 2 f$, and $3 f$, respectively. Moreover, note that by restricting ourselves in (15) to only these harmonics, we neglect all the higher (than $3 f$ ) ones in the calculations - as it has been done in papers [1]-[10].

Introducing (15) into the truncated series of (10) gives

$$
\hat{y}(t)=y^{(1)}(t)+y^{(2)}(t)+y^{(3)}(t)
$$

with

$$
\begin{gathered}
y^{(1)}(t)=H^{(1)}(f) X_{f} e^{j 2 \pi f t}+H^{(1)}(2 f) X_{2 f} e^{j 2 \pi(2 f) t}+ \\
+H^{(1)}(3 f) X_{3 f} e^{j 2 \pi(3 f) t}
\end{gathered}
$$

$$
\begin{gathered}
y^{(2)}(t)=H^{(2)}(f, f) X_{f}^{2} e^{j 2 \pi(2 f) t}+2 H^{(2)}(f, 2 f) \cdot \\
\cdot X_{f} X_{2 f} e^{j 2 \pi(3 f) t}+\left[2 H^{(2)}(f, 3 f) X_{f} X_{3 f}+\right. \\
\left.+H^{(2)}(2 f, 2 f) X_{2 f}^{2}\right] e^{j 2 \pi(4 f) t}+\ldots \\
y^{(3)}(t)=H^{(3)}(f, f, f) X_{f}^{3} e^{j 2 \pi(3 f) t}+ \\
+3 H^{(3)}(f, f, 2 f) X_{f}^{2} X_{2 f} e^{j 2 \pi(4 f) t}+\ldots
\end{gathered}
$$

where it has been assumed that the nonlinear transfer functions are symmetric ones (that is, for example, $H^{(2)}(f, 2 f)=H^{(2)}(2 f, f)$, and so on; for more details regarding this issue, see, for instance, [13]).

Observe that the resulting signal given by (16) contains now new harmonics $4 f$ and higher ones, which do not occur in the input signal (15). This is opposite to the previous case of (11), where no new harmonics were generated. The signal components at the aforementioned harmonics are neglected in the method presented in [1]-[10]. In other words, the following expressions are used therein instead of (16c) and (16d)

$$
\begin{gathered}
\tilde{y}^{(2)}(t)=H^{(2)}(f, f) X_{f}^{2} e^{j 2 \pi(2 f) t}+2 H^{(2)}(f, 2 f) . \\
\cdot X_{f} X_{2 f} e^{j 2 \pi(3 f) t}
\end{gathered}
$$

and

$$
\tilde{y}^{(3)}(t)=H^{(3)}(f, f, f) X_{f}^{3} e^{j 2 \pi(3 f) t} .
$$

The symbol " $\sim$ " in (17a) and (17b) means operation of neglecting the higher harmonics than $3 f$ in the corresponding partial responses $y^{(i)}(t)$ (here $i=2$ or 3 ).

Equations (16a) - (16d) describe and define at the same time correctly the operations that are understood under the operator "o" introduced in a descriptive way in [2]. The definition of the operator "o" in [2] is incorrect. We show this using the same example as that which was given by S. O. Cannizarro,
G. Palumbo, and S. Pennisi in [2] (beneath equations (11) and (12) therein) for illustration of their descriptive definition of "o". To this end, let us formulate the aforementioned example using our notation as: calculate $\hat{y}(t)=y^{(1)}(t)+y^{(2)}(t)$ for the input signal $x(t)=X_{f} e^{j 2 \pi f t}+X_{2 f} e^{j 2 \pi(2 f) t}$. Note that to get a solution to this problem, we can use expressions (16b), (16c), and (16d) already derived by substitution $X_{3 f} \equiv 0$ and $H^{(3)}(\cdot, \cdot, \cdot) \equiv 0$ therein. This leads to

$$
\begin{gathered}
\hat{y}_{e x}(t)=X_{f} H^{(1)}(f) e^{j 2 \pi f t}+\left[X_{2 f} H^{(1)}(2 f)+\right. \\
\left.+X_{f}^{2} H^{(2)}(f, f)\right] e^{j 2 \pi(2 f) t}+2 X_{f} X_{2 f} H^{(2)}(f, 2 f) . \\
\cdot e^{j 2 \pi(3 f) t}+X_{2 f}^{2} H^{(2)}(2 f, 2 f) e^{j 2 \pi(4 f) t} .
\end{gathered}
$$

Evidently, the multipliers of the complex function $e^{j 2 \pi(3 f) t}$ in (18) and in the corresponding expression derived in [2] differ from each other.

For those who want to avoid the use of Volterra series in nonlinear analysis exploiting phasors, let us explain the problem discussed in the above example in another way. So to this end, let us consider a simple nonlinear capacitor described by the relation

$$
i_{C}(t)=\frac{d q(t)}{d t}=\frac{d}{d t}\left(a_{1} v_{C}(t)+a_{2}\left(v_{C}(t)\right)^{2}\right)
$$

where $i_{C}, q$, and $v_{C}$ are the current flowing through, the charge on, and the voltage across the capacitor, respectively. The assumed dependence of $q$ upon $v_{C}$ in (19a) is $q=a_{1} v_{C}+a_{2} v_{C}^{2}$ with $a_{1}$ and $a_{2}$ being constant coefficients in this polynomial expansion ( $a_{1}=C$ is called the capacitor capacitance $C$ ). Further, assume that the voltage across the capacitor is given by

$$
v_{C}(t)=X_{f} e^{j 2 \pi f t}+X_{2 j} e^{j 2 \pi(2 f) t}
$$

Introducing (19b) into (19a) gives

$$
\begin{gathered}
i_{C}(t)=X_{f} j 2 \pi f a_{1} e^{j 2 \pi f t}+\left[X_{2 f} j 2 \pi(2 f) a_{1}+\right. \\
\left.+X_{f}^{2} j 2 \pi(2 f) a_{2}\right] e^{j 2 \pi(2 f) t}+2 X_{f} X_{2 f} j 2 \pi(3 f) a_{2} . \\
\cdot e^{j 2 \pi(3 f) t}+X_{2 f}^{2} j 2 \pi(4 f) a_{2} e^{j 2 \pi(4 f) t} .
\end{gathered}
$$

Compare now the coefficient $j 2 \pi(3 f) a_{2}$ multiplying $2 X_{f} X_{2 f} e^{j 2 \pi(3 f) t}$ in (20) with the corresponding coefficient derived in [2], which will be equal to $\left[j 2 \pi(2 f) a_{2}+j 2 \pi(4 f) a_{2}\right]=j 2 \pi(2 f+4 f) a_{2}=j 2 \pi(6 f) a_{2}$ in the case of our capacitor. Because evidently $j 2 \pi(3 f) a_{2} \neq j 2 \pi(6 f) a_{2}$, this shows that the definition of the operator "o" in [2] is incorrect.

By the way, observe that we can identify the corresponding (nonlinear) non-rotating phasors with the nonlinear transfer functions describing our nonlinear capacitor considered in the frequency domain - by comparison of (18) with (20). Then, we get

$$
H^{(1)}(f)=j 2 \pi f a_{1}
$$

and

$$
H^{(2)}(f, f)=j 2 \pi(f+f) a_{2}=j 2 \pi(2 f) a_{2} .
$$

Further, note in (21a) that the function $H^{(2)}(f, f)$ of two variables is described by an expression in which these two 
variables do not occur "separately", but they are added to each other. This property can be generalized for mildly nonlinear RLC circuits including nonlinear amplifiers - see for example [13], [18].

Obviously, the phasors can be introduced into the nonlinear analysis of ac circuits without referring to such notions as the Volterra series and multidimensional Fourier transforms. Then, similarly as in the usual phasor analysis of linear ac circuits (in form as developed by Ch. P. Steinmetz), we do not need to refer to the convolution integral and Fourier transform. In this case, we exploit the so-called constitutive relations [19] defining the basic circuit elements (not any other descriptions of circuit elements!) Now, to illustrate this point in more detail, let us consider once again an example of the nonlinear capacitor described by the relation (19a) defining the constitutive equation of this circuit element. So, according to the basic principle of the phasor method, we apply $v_{C}(t)=X_{f} e^{j 2 \pi f t}$ in (19a). This results in

$$
\begin{aligned}
i_{C}(t) & =j 2 \pi f a_{1} X_{f} e^{j 2 \pi f t}+j 2 \pi(2 f) a_{2} X_{f}^{2} e^{j 2 \pi(2 f) t}= \\
& =j 2 \pi f a_{1} v_{C}(t)+j 2 \pi(2 f) a_{2}\left(v_{C}(t)\right)^{2}= \\
& =Y_{C}^{(1, a)}(f) v_{C}(t)+Y_{C}^{(2, a)}(2 f)\left(v_{C}(t)\right)^{2}
\end{aligned}
$$

where $Y_{C}^{(1, a)}(f)=j 2 \pi f a_{1}$ and $Y_{C}^{(2, a)}(2 f)=j 2 \pi(2 f) a_{2}$ are the non-rotating phasors constituting the coefficients of the rotating phasors $Y_{C}^{(1, a)}(f) v_{C}(t)$ and $Y_{C}^{(2, a)}(2 f)\left(v_{C}(t)\right)^{2}$, respectively. The latter ones rotate with the corresponding frequencies $f$ and $2 f$. So, because of the above facts, we can name $Y_{C}^{(1, a)}(f)$ and $Y_{C}^{(2, a)}(2 f)$ the capacitor phasors of the first and second order, accordingly. Moreover, we put additionally the letter " $a$ " in the superscripts of these phasors to indicate the fact that they are calculated for the input signal being a single complex harmonic function $X_{f} e^{j 2 \pi f t}$. Thereby, we distinguish this case from another one, "b", standing for the calculations with the circuit input signal consisting of a sum of complex harmonic signals at frequencies $f, 2 f$, and $3 f$.

Note that, as it was already pointed out, the case " $b$ " takes place, when a circuit element is not directly driven by an input signal applied to the circuit. That is it is an internal nonlinear circuit element, and therefore, driven by a signal having form of a sum of harmonics of the circuit input signal (including the first one). In what follows now, we explain this case on an example of our nonlinear capacitor discussed just before, restricting ourselves to taking into account only two first harmonics as assumed in (19b).

The result of substitution of (19b) into the capacitor explicit constitutive relation (19a) is given by (20). Observe that the signal harmonics $3 f$ and $4 f$ occur in (20), but they are absent in the signal given by (19b). Therefore, we truncate (neglect) them (this is a standard operation performed in the method presented in [1]-[10]). As a result, we get

$$
\begin{aligned}
& \tilde{i}_{C}(t)=j 2 \pi f a_{1} X_{f} e^{j 2 \pi f t}+\left[j 2 \pi(2 f) a_{1} X_{2 f}+\right. \\
& \left.+j 2 \pi(2 f) a_{2} X_{f}^{2}\right] e^{j 2 \pi(2 f) t}=j 2 \pi f a_{1} X_{f} e^{j 2 \pi f t}+ \\
& +\left[j 2 \pi(2 f) a_{1} \frac{X_{2 f}}{X_{f}^{2}}+j 2 \pi(2 f) a_{2}\right]\left(X_{f} e^{j 2 \pi f t}\right)^{2}=
\end{aligned}
$$

$$
=Y_{C}^{(1, b)}(f)\left(X_{f} e^{j 2 \pi f t}\right)+Y_{C}^{(2, b)}(2 f)\left(X_{f} e^{j 2 \pi f t}\right)^{2}
$$

where the non-rotating phasors $Y_{C}^{(1, b)}(f)$ and $Y_{C}^{(2, b)}(2 f)$ are the counterparts in this case "b" of the phasors $Y_{C}^{(1, a)}(f)$ and $Y_{C}^{(2, a)}(2 f)$ occurring in (22), respectively. Moreover, comparison of (22) with (23) shows that the following relations hold

$$
Y_{C}^{(1, b)}(f)=Y_{C}^{(1, a)}(f)=j 2 \pi f a_{1}
$$

and

$$
\begin{gathered}
Y_{C}^{(2, b)}(2 f)=Y_{C}^{(1, a)}(2 f) \frac{X_{2 f}}{X_{f}^{2}}+Y_{C}^{(2, a)}(2 f)= \\
=j 2 \pi(2 f) a_{1} \frac{X_{2 f}}{X_{f}^{2}}+j 2 \pi(2 f) a_{2} .
\end{gathered}
$$

Concluding and generalizing the above example, we say that having a unique constitutive relation for a given nonlinear circuit element, we derive from it two different phasor-based descriptions (compare (22) with (23) and see (24b)) for the nonlinear analysis in the frequency domain. Their usage depends upon the place where the given element occurs in the circuit - with respect to the placement of the circuit input signal.

By the way, observe also that the derivations presented up to now show that not only the use of phasors but also such the tools as Volterra series and multidimensional Fourier transforms make the nonlinear analysis better understandable and more transparent.

The descriptions derived in (22) and (23), and called the input-output and in-network representations, respectively, are illustrated in Fig. 1.

Finally, note that Fig. 1, and eqs. (22) and (23) show clearly that the input-output representation given by (22) cannot be treated as a constitutive equation for the derivation of the innetwork representation expressed by (23).

After finishing discussion of the phasor representations of nonlinear elements, let us consider now formulation of circuit equations with the use of these descriptions. There exist many possibilities for such formulations; basically, they can be put into two groups: on one side those using matrix formalism, as for example, exploiting admittance, impedance, or (mixed) modified nodal formulation [18], [20], and on the other side such that apply operators working on signals and graphs. The method presented in [1]-[10] uses the second approach, and in what follows, we refer to it.

First, observe that the form of the non-rotating phasors as $Y_{C}^{(1, a)}$ and $Y_{C}^{(2, a)}$ in (22), and even the complex-amplitude dependent $Y_{C}^{(2, b)}$ in (23) are not suitable for formulation of the circuit equations. Exploiting once again the nonlinear capacitor example, we show how equations (22) and (23) should be reformulated for the aforementioned needs, namely as

$$
i_{C}(t)=I_{C}^{(1, a)} e^{j 2 \pi f t}+I_{C}^{(2, a)} e^{j 2 \pi(2 f) t}
$$

in the case of (22), with the non-rotating phasors dependent now upon the signal complex amplitudes (that is having the form of those of first type - according to the definition introduced in Section II). So, they will 


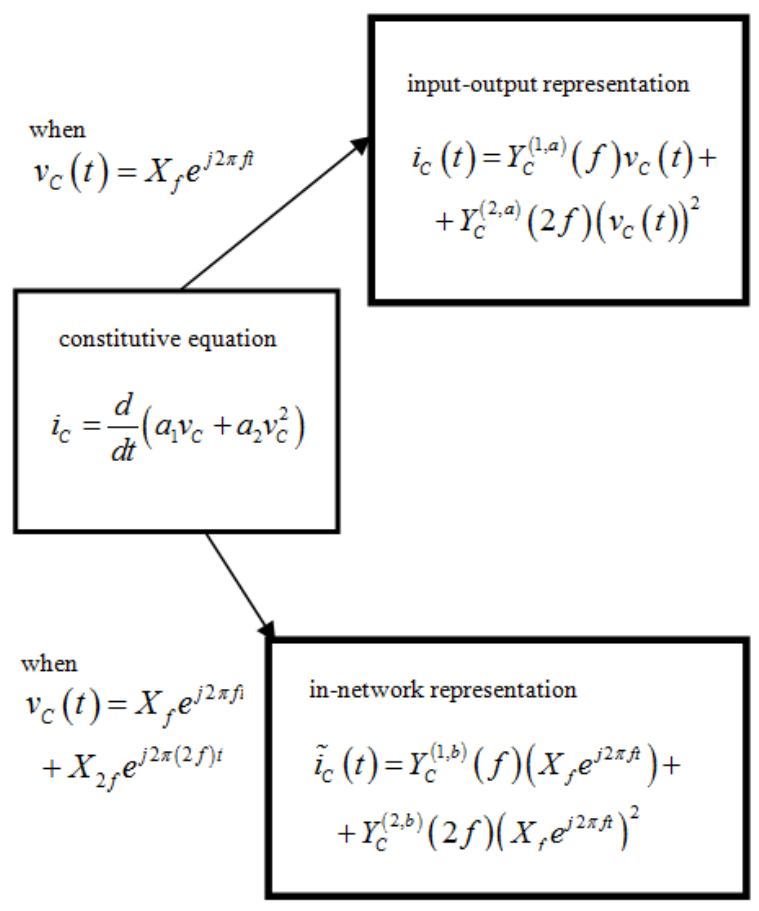

Fig. 1. Illustration of the derivation of the input-output (eq. (22)) and innetwork (eq. (23)) representations from the explicit constitutive equation of a nonlinear capacitor given by (19a).

be then defined as $I_{C}^{(1, a)}=j 2 \pi f a_{1} X_{f}=Y_{C}^{(1, a)}(f) X_{f}$ and $I_{C}^{(2, a)}=j 2 \pi(2 f) a_{2} X_{f}^{2}=Y_{C}^{(2, a)}(2 f) X_{f}^{2}$. And, similarly in the case of (23), we will have

$$
\tilde{i}_{C}(t)=I_{C}^{(1, b)} e^{j 2 \pi f t}+I_{C}^{(2, b)} e^{j 2 \pi(2 f) t}
$$

with the non-rotating phasors reformulated for the case "b", given by $I_{C}^{(1, b)}=j 2 \pi f a_{1} X_{f}=Y_{C}^{(1, b)}(f) X_{f}$ and $I_{C}^{(2, b)}=j 2 \pi(2 f)\left[a_{1} X_{2 f}+a_{2} X_{f}^{2}\right]=Y_{C}^{(2, b)}(2 f) X_{f}^{2}$. At this point, we remind also that the number 1 in the superscript of $I_{C}^{(1, b)}$, and the number 2 in the superscript of $I_{C}^{(2, b)}$ stay for the orders of these phasors. (Caution: Note that, according to the definitions introduced, the type and order of a phasor do mean two different things!)

Now, to illustrate the basic principles of the "operatorgraph" method of formulation of circuit equations with the use of non-rotating phasors of the first and second type, consider a scheme of a simple nonlinear circuit shown in Fig. 2, which consists of a nonlinear capacitor (NC) and a nonlinear conductor (NG) connected in parallel and driven by an independent current source $I_{S} e^{j 2 \pi f t}$.

The currents $i_{i}(t), i_{C}(t)$ and $i_{G}(t)$, and the voltage $v(t)=v_{C}(t)=v_{G}(t)$ are defined in Fig. 2. And, let the circuit input and output signals be $i_{i}(t)$ and $i_{G}(t)$, respectively. Further, the balance of currents at the circuit node expresses as

$$
i_{i}(t)=i_{C}(t)+i_{G}(t)
$$

Because the circuit analysed is nonlinear, the currents $i_{C}(t)$ and $i_{G}(t)$ possess also the second and third harmonic components. Therefore, using (26), we write

$$
I_{S} e^{j 2 \pi f t}+0 \cdot e^{j 2 \pi(2 f) t}+0 \cdot e^{j 2 \pi(3 f) t}=
$$

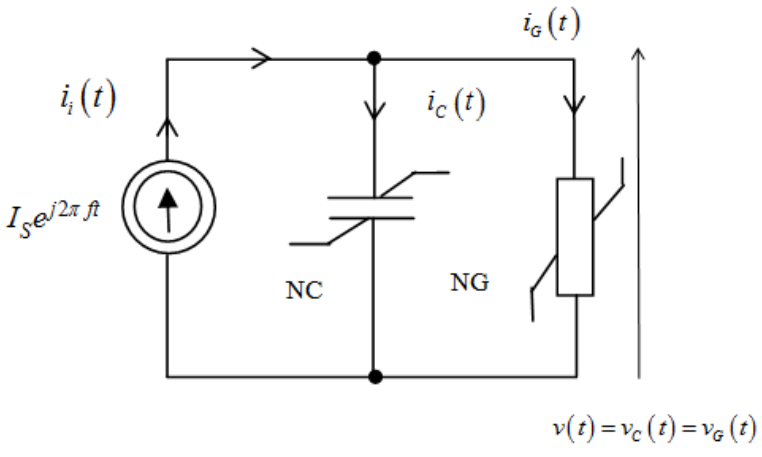

Fig. 2. A simple nonlinear circuit consisting of two nonlinear elements: capacitor and conductor connected in parallel.

$$
\begin{aligned}
= & I_{C f} e^{j 2 \pi f t}+I_{C 2 f} e^{j 2 \pi(2 f) t}+I_{C 3 f} e^{j 2 \pi(3 f) t}+ \\
& +I_{G f} e^{j 2 \pi f t}+I_{G 2 f} e^{j 2 \pi(2 f) t}+I_{G 3 f} e^{j 2 \pi(3 f) t}
\end{aligned}
$$

where $I_{C f}, I_{C 2 f}, I_{C 3 f}$ mean the amplitudes of the first, second, and third harmonic component, respectively, of the current $i_{C}(t)$. The similar regards $I_{G f}, I_{G 2 f}$, and $I_{G 3 f}$, being the corresponding harmonic amplitudes in $i_{G}(t)$. (Note that the notations $I_{C f}, I_{G f}, I_{C 2 f}, I_{G 2 f}, I_{C 3 f}$ and $I_{G 3 f}$ correspond to $X_{f}, X_{2 f}$ and $X_{3 f}$ used in a more general context in the previous derivations.)

Equating then to each other the amplitudes standing by the corresponding exponents $e^{j 2 \pi f t}, e^{j 2 \pi(2 f) t}$ and $e^{j 2 \pi(3 f) t}$ on both sides of (27), we get

$$
\begin{gathered}
I_{S}=I_{C f}+I_{G f} \\
0=I_{C 2 f}+I_{G 2 f} \\
0=I_{C 3 f}+I_{G 3 f} .
\end{gathered}
$$

Note that eqs. (28a-c) describe the balance of harmonics at the node of circuit of Fig. 2 - in terms of amplitudes of these harmonics.

In the next step, note that the amplitudes of harmonics occurring in eqs. (28a-c) can be expressed by the non-rotating phasors of the first type associated with the nonlinear capacitor and nonlinear conductor. Because of the fact that the voltage $v(t)$, being the terminal input signal for $\mathrm{NC}$ and $\mathrm{NG}$, is not equal to the circuit input signal $I_{S} e^{j 2 \pi f t}$, we will use the second rule derived previously (denoted as the case " $b$ ") for these phasors. So, applying this, we can rewrite eqs. (28a-c) as

$$
\begin{gathered}
I_{S}=I_{C}^{(1, b)}+I_{G}^{(1, b)} \\
0=I_{C}^{(2, b)}+I_{G}^{(2, b)} \\
0=I_{C}^{(3, b)}+I_{G}^{(3, b)} .
\end{gathered}
$$

At this point, we remind that the numbers 1, 2, and 3 denoting the corresponding orders of the phasors in eqs. (28a-c) mean that these phasors stand (as amplitudes) by the complex functions $e^{j 2 \pi f t}, e^{j 2 \pi(2 f) t}$ and $e^{j 2 \pi(3 f) t}$, respectively. 
In an example of circuit of Fig. 2, we assume that the element $\mathrm{NC}$ is described by the constitutive equation (19a) and the element NG by the following one

$$
i_{G}=g_{1} v_{G}+g_{2} v_{G}^{2} .
$$

In both eqs. (19a) and (30), the first and second order (only) nonlinearities are incorporated in.

Adding now the additional component $X_{3 f} e^{j 2 \pi(3 f) t}$ in (19b), renaming the amplitudes $V_{f}=X_{f}, V_{2 f}=X_{2 f}$, and $V_{3 f}=X_{3 f}$ in the resulting equation, introducing it afterwards in (19a), and finally restricting the procedure to consideration of only the first three harmonics, we arrive at

$$
\begin{gathered}
\tilde{i}_{C}(t)=j 2 \pi f a_{1} V_{f} e^{j 2 \pi f t}+\left[j 2 \pi(2 f) a_{1} V_{2 f}+\right. \\
\left.+j 2 \pi(2 f) a_{2} V_{f}^{2}\right] e^{j 2 \pi(2 f) t}+\left[j 2 \pi(3 f) a_{1} V_{3 f}+\right. \\
\left.+2 j 2 \pi(3 f) a_{2} V_{f} V_{2 f}\right] e^{j 2 \pi(3 f) t}
\end{gathered}
$$

Note that using the non-rotating phasors of the second type we can rewritre (31) as

$$
\begin{gathered}
\tilde{i}_{C}(t)=Y_{C}^{(1)}(f) V_{f} e^{j 2 \pi f t}+\left[Y_{C}^{(1)}(2 f) V_{2 f}+\right. \\
\left.+Y_{C}^{(2)}(2 f) V_{f}^{2}\right] e^{j 2 \pi(2 f) t}+\left[Y_{C}^{(1)}(3 f) V_{3 f}+\right. \\
\left.+2 Y_{C}^{(2)}(3 f) V_{f} V_{2 f}\right] e^{j 2 \pi(3 f) t}
\end{gathered}
$$

with

$$
Y_{C}^{(1)}(f)=Y_{C}^{(1, a)}(f)=j 2 \pi f a_{1}
$$

and

$$
Y_{C}^{(2)}(2 f)=Y_{C}^{(2, a)}(f)=j 2 \pi(2 f) a_{2}
$$

$Y_{C}^{(1)}(2 f), Y_{C}^{(1)}(3 f)$ and $Y_{C}^{(2)}(3 f)$ in (32a) are defined by (32b) and (32c) with another frequency arguments $2 f, 3 f$, and $3 f$, respectively. Note that we have now dropped the indication of the case "a" in $Y_{C}^{(1, a)}$ and $Y_{C}^{(2, a)}$, shortened to $Y_{C}^{(1)}$ and $Y_{C}^{(2)}$, for simplicity. Moreover, eqs. (23) and (24b) show that introduction of a special kind of non-rotating phasors of second type for the second case " $b$ " is useless because, as for example $Y_{C}^{(2, b)}$ in (24b) is still dependent upon signal amplitudes. We will use the above simplified notation consequently in what follows.

Eq. (31) or (32a) allow us to find the expressions for the non-rotating phasors of the first type versus the phasors of the second type for the NC in Fig. 2. So, we identify in this case

$$
\begin{gathered}
I_{C}^{(1, b)}=j 2 \pi f a_{1} V_{f}=Y_{C}^{(1)}(f) V_{f} \\
I_{C}^{(2, b)}=j 2 \pi(2 f) a_{1} V_{2 f}+j 2 \pi(2 f) a_{2} V_{f}^{2}= \\
=Y_{C}^{(1)}(2 f) V_{2 f}+Y_{C}^{(2)}(2 f) V_{f}^{2} \\
I_{C}^{(3, b)}=j 2 \pi(3 f) a_{1} V_{3 f}+2 j 2 \pi(3 f) a_{2} V_{f} V_{2 f}= \\
=Y_{C}^{(1)}(3 f) V_{3 f}+2 Y_{C}^{(2)}(3 f) V_{f} V_{2 f} .
\end{gathered}
$$

Proceeding now for NG in Fig. 1 similarly as for NC above, we arrive at the following counterparts of (31), (32a-c), and $(33 \mathrm{a}-\mathrm{c})$

$$
\tilde{i}_{G}(t)=g_{1} V_{f} e^{j 2 \pi f t}+\left[g_{1} V_{2 f}+g_{2} V_{f}^{2}\right] e^{j 2 \pi(2 f) t}+
$$

$$
\begin{gathered}
+\left[g_{1} V_{3 f}+2 g_{2} V_{f} V_{2 f}\right] e^{j 2 \pi(3 f) t} \\
\tilde{i}_{G}(t)=Y_{G}^{(1)}(f) V_{f} e^{j 2 \pi f t}+\left[Y_{G}^{(1)}(2 f) V_{2 f}+\right. \\
\left.+Y_{G}^{(2)}(2 f) V_{f}^{2}\right] e^{j 2 \pi(2 f) t}+\left[Y_{G}^{(1)}(3 f) V_{3 f}+\right. \\
\left.+2 Y_{G}^{(2)}(3 f) V_{f} V_{2 f}\right] e^{j 2 \pi(3 f) t}
\end{gathered}
$$

with

$$
Y_{G}^{(1)}(\cdot)=Y_{G}^{(1, a)}(\cdot)=g_{1}
$$

and

$$
Y_{G}^{(2)}(\cdot)=Y_{G}^{(2, a)}(\cdot)=g_{2}
$$

and

$$
\begin{gathered}
I_{G}^{(1, b)}=g_{1} V_{f}=Y_{G}^{(1)}(f) V_{f} \\
I_{G}^{(2, b)}=g_{1} V_{2 f}+g_{2} V_{f}^{2}=Y_{G}^{(1)}(2 f) V_{2 f}+Y_{G}^{(2)}(2 f) V_{f}^{2} \\
I_{G}^{(3, b)}=g_{1} V_{3 f}+2 g_{2} V_{f} V_{2 f}=Y_{G}^{(1)}(3 f) V_{3 f}+ \\
+2 Y_{G}^{(2)}(3 f) V_{f} V_{2 f} .
\end{gathered}
$$

Substituting then (33a-c) and (36a-c) into (29a-c), and solving for $V_{f}, V_{2 f}$, and $V_{3 f}$, we obtain

$$
\begin{gathered}
V_{f}=\frac{I_{S}}{g_{1}+j 2 \pi f a_{1}} \\
V_{2 f}=-I_{S}^{2} \frac{g_{2}+j 2 \pi(2 f) a_{2}}{\left(g_{1}+j 2 \pi(2 f) a_{1}\right)\left(g_{1}+j 2 \pi f a_{1}\right)^{2}} \\
V_{3 f}=I_{S}^{3} \frac{2\left(g_{2}+j 2 \pi(2 f) a_{2}\right)\left(g_{2}+j 2 \pi(3 f) a_{2}\right)}{\left(g_{1}+j 2 \pi(3 f) a_{1}\right)\left(g_{1}+j 2 \pi(2 f) a_{1}\right)\left(g_{1}+j 2 \pi f a_{1}\right)^{2}}
\end{gathered}
$$

Finally, using $V_{f}=V_{C f}=V_{G f}, V_{2 f}=V_{C 2 f}=V_{G 2 f}$, and $V_{3 f}=V_{C 3 f}=V_{G 3 f}$, given by (37a-c) in (33a-c) and (36a-c), we can express, respectively, the amplitudes of the first three harmonic components of the currents $i_{C}(t)$ (i.e. $I_{C f}, I_{C 2 f}$, and $\left.I_{C 3 f}\right)$ and $i_{G}(t)$ (i.e. $I_{G f}, I_{G 2 f}$, and $I_{G 3 f}$ ) occurring in Fig. 2 versus the amplitude $I_{S}$ of the input current signal. Moreover, it follows from the form of $V_{f}, V_{2 f}$, and $V_{3 f}$ in $(37 \mathrm{a}-\mathrm{c})$ that they are non-rotating phasors of the first type for the case "a" with respect to the input signal $I_{S} e^{j 2 \pi f t}$. That is we write $V_{f}=V^{(1, a)}, V_{2 f}=V^{(2, a)}$, and $V_{3 f}=V^{(3, a)}$. At the same time, they constitute the non-rotating phasors of the first type for the case "b", too. However, in the latter case in other equations having the form as, for example, eqs. (33a-c), they play such a role with respect to the terminal signals $\tilde{i}_{C}(t)$ and $\tilde{i}_{G}(t)$ consisting of the first three harmonic components having the amplitudes, say, $I_{C f}, I_{C 2 f}, I_{C 3 f}$ and $I_{G f}, I_{G 2 f}, I_{G 3 f}$, respectively. This can be easily checked using eqs. (33a-c) together with the relation inverting (19a) and (36a-c) together with the relation inverting (30), respectively.

The non-rotating phasors of the second type can be used in the nonlinear phasor analysis, similarly as in the linear case, to construct equivalent schemes of parts of a circuit consisting of interconnected basic circuit elements. And we illustrate now this point, once again, on an example of the circuit of Fig. 2. For a parallel connection of $\mathrm{NC}$ and $\mathrm{NG}$ in this figure, we build an equivalent scheme. So, then, Fig. 2 can be redrawn in a simplified form as shown in Fig. 3. 


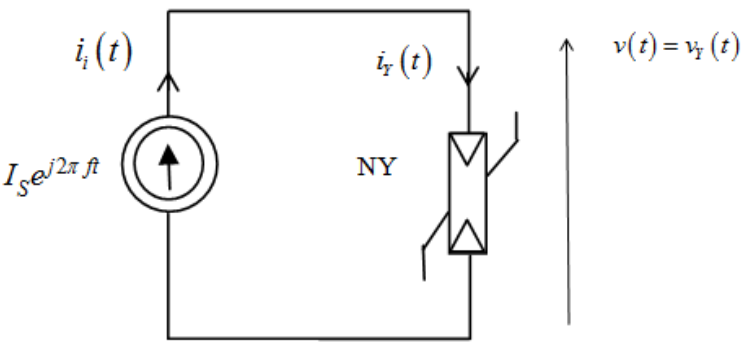

Fig. 3. A simplified form of the circuit of Fig. 2 with the parallel connection of $\mathrm{NC}$ and $\mathrm{NG}$ replaced by an equivalent element $\mathrm{NY}$.

Observe that the amplitudes of the first three harmonic components $I_{Y}^{(1, b)}, I_{Y}^{(2, b)}$, and $I_{Y}^{(3, b)}$ of the current $i_{Y}(t)$ flowing through the equivalent element NY (nonlinear admittance) in Fig. 3 can be expressed, using (33a-c) and (36a-c), as

$$
\begin{gathered}
I_{Y}^{(1, b)}=I_{C}^{(1, b)}+I_{G}^{(1, b)}=\left(g_{1}+j 2 \pi f a_{1}\right) V_{f} \\
I_{Y}^{(2, b)}=I_{C}^{(2, b)}+I_{G}^{(2, b)}=\left(g_{1}+j 2 \pi(2 f) a_{1}\right) V_{2 f}+ \\
\quad+\left(g_{2}+j 2 \pi(2 f) a_{2}\right) V_{f}^{2} \\
I_{Y}^{(3, b)}=I_{C}^{(3, b)}+I_{G}^{(3, b)}=\left(g_{1}+j 2 \pi(3 f) a_{1}\right) V_{3 f}+ \\
+2\left(g_{2}+j 2 \pi(3 f) a_{2}\right) V_{f} V_{2 f} .
\end{gathered}
$$

Comparison of eqs. (38a-c) with eqs. (33a-c) or (36a-c) shows that the expressions

$$
Y_{Y}^{(1)}(f)=g_{1}+j 2 \pi f a_{1}=Y_{G}^{(1)}(f)+Y_{C}^{(1)}(f)
$$

and

$$
Y_{Y}^{(1)}(2 f)=g_{2}+j 2 \pi(2 f) a_{2}=Y_{G}^{(2)}(2 f)+Y_{C}^{(2)}(2 f)
$$

play analogous role as $Y_{G}^{(1)}(f)$ and $Y_{G}^{(2)}(2 f)$ or $Y_{C}^{(1)}(f)$ and $Y_{C}^{(2)}(2 f)$ in (33a-c) or (36a-c), respectively. These expressions describe fully the equivalent nonlinear element NY. Furthermore, they are constructed using exclusively the phasors of the second type describing NG and NC. This is their characteristic feature.

Observe that the non-rotating phasors of the second type $Y_{G}^{(3)}(3 f)$ and $Y_{C}^{(3)}(3 f)$ are equal to zero in the example considered, and consequently $Y_{Y}^{(3)}(3 f) \equiv 0$. This follows from the fact that the coefficients: $a_{3} \equiv 0$ in (19a) and $g_{3} \equiv 0$ in (30); that is the highest order of nonlinearity in NC and NG of our example is restricted to two. Consequently, the highest order of the nonzero non-rotating phasors $Y_{G}^{(i)}(\cdot)$ and $Y_{C}^{(i)}(\cdot)$, $i=1,2,3, \ldots$, is equal to two.

The above fact can be generalized as follows: An order of a given non-rotating phasor of the second type is equal to the order of nonlinearity described by this phasor. Opposite to this, from the previous derivations, it follows that the orders of the non-rotating phasors of the first type provide other information. They are tightly associated with the orders of harmonics in such a way that the non-rotating phasor of the first type and of the $i$-th order must be multiplied by $e^{j 2 \pi(i f) t}$ to give the complex harmonic signal of frequency if.

\section{SUMMARY}

In this paper, a thorough and mathematically rigorous treatment of the phasor use in nonlinear analysis has been presented. This fills the lack of such a theory in the literature; thanks to it some partly incorrect derivations occurring in papers [1]-[10] can be now corrected. The characteristic features of this theory are as follows:

1) The fact that we can distinguish between two kinds of non-rotating phasors: dependent and independent of amplitudes of the signal harmonic components and/or of their products allows us to interpret their orders in the nonlinear analysis as associated strictly with the corresponding orders of harmonics and orders of nonlinearity, respectively. We refer to these phasors as to the first and second type ones, accordingly.

2) Two means of "transferring harmonics" from a terminal (port) input to terminal (port) output or from a whole circuit input to its output refer exclusively to the nonrotating phasors of the first type. These two means are characterized by different calculation rules, depicted here as the cases "a" and "b".

3) Balance of harmonics is carried out at nodes of a circuit or of its representing graph with the use of non-rotating phasors of the first type, as for example shown in equations $(29 a-c)$.

4) We express phasors of the first type versus phasors of the second type. We use then, consequently, a certain very important convention; we explain it on an example: Take into account $Y_{C}^{(2)}(3 f)$. In this phasor, the number 2 means the order of nonlinearity dealt with, but the argument $3 f$ denotes harmonic frequency for which this phasor is calculated.

5) As shown, the balance of harmonics relies upon the use of non-rotating phasors of the first type. But calculation of the equivalent schemes of the interconnected circuit nonlinear basic elements needs the use of only nonrotating phasors of the second type.

\section{REFERENCES}

[1] G. Palumbo and S. Pennisi, "High-frequency harmonic distortion in feedback amplifiers: analysis and applications," IEEE Transactions on Circuits and Systems-I: Fundamental Theory and Applications, vol. 50, pp. 328-340, March 2003.

[2] S. O. Cannizzaro, G. Palumbo, and S. Pennisi, "Effects of nonlinear feedback in the frequency domain," IEEE Transactions on Circuits and Systems-I: Regular Papers, vol. 53, pp. 225-234, February 2006.

[3] —, "Distortion analysis of Miller-compensated three-stage amplifiers," IEEE Transactions on Circuits and Systems-I: Regular Papers, vol. 53, pp. 961-976, May 2006.

[4] G. Palumbo, M. Pennisi, and S. Pennisi, "Analysis and evaluation of harmonic distortion in the tunnel diode oscillator," in Proceedings of ISCAS'06, Island of Kos, May 2006, pp. 5135-5138.

[5] — - "Analysis and evaluation of harmonic distortion in the Hartley oscillator," in Proceedings of NOLTA 2006, Bologna, September 2006, pp. 643-646.

[6] - "Analysis of harmonic distortion in the Colpitts oscillator," in Proceedings of ICECS'06, Nice, December 2006, pp. 196-199.

[7] _ - "Distortion analysis in the frequency domain of a Gm-C biquad filter," in Proceedings of ECCTD'07, Seville, August 2007, pp. 212-215.

[8] S. O. Cannizzaro, G. Palumbo, and S. Pennisi, "An approach to model high-frequency distortion in negative-feedback amplifiers," Journal of Circuit Theory and Applications, vol. 36, pp. 3-18, January 2008. 
[9] G. Palumbo, M. Pennisi, and S. Pennisi, "Wien-type oscillators: evaluation and optimization of harmonic distortion," IEEE Transastions on Circuits and Systems-II: Express Briefs, vol. 55, pp. 628-632, July 2008.

[10] _ "Miller theorem for weakly nonlinear feedback circuits and application to CE amplifier," IEEE Transastions on Circuits and Systems-II: Express Briefs, vol. 55, pp. 991-995, October 2008.

[11] S. Narayanan, "Application of Volterra series to intermodulation distortion analysis of transistor feedback amplifiers," IEEE Transactions on Circuit Theory, vol. CT-17, pp. 518-527, November 1970.

[12] E. Bedrosian and S. O. Rice, "The output properties of Volterra systems (nonlinear systems with memory) driven by harmonic and Gaussian inputs," Proceedings of the IEEE, vol. 59, pp. 1688-1707, December 1971.

[13] J. J. Bussgang, L. Ehrman, and J. W. Graham, "Analysis of nonlinear systems with multiple inputs," Proceedings of the IEEE, vol. 62, pp. 1088-1119, August 1971.

[14] C. P. Steinmetz, "Theory and calculation of a. c. phenomena," 1897 New York.
[15] B. Konorski, Podstawy Elektrotechniki. Warszawa: PWN, 1961, (in Polish).

[16] S. Boyd and L. O. Chua, "Fading memory and the problem of approximating nonlinear operators with Volterra series," IEEE Transactions on Circuits and Systems, vol. CAS-32, pp. 1150-1161, November 1985.

[17] I. W. Sandberg, "Causality and the impulse response scandal," IEEE Transactions on Circuits and Systems - I: Fundamental Theory and Applications, vol. 50, pp. 810-811, 2003.

[18] A. Borys, "A simplified analysis of nonlinear distortion in analog electronic circuits using the Volterra-Wiener series," Scientia Electrica, vol. 30, no. 3, pp. 78-103, 1984

[19] L. O. Chua, "Device modeling via basic nonlinear circuit elements," IEEE Transactions on Circuits Systems, vol. CAS-27, pp. 1014-1044, November 1980

[20] A. Borys, "Exploiting admittance formalism in the nonlinear analysis," Zesz. Nauk. WSInf: Teoria i Zastosowania Informatyki, vol. 8, no. 2, pp. 17-39, 2009. 